\title{
La dimensión social del actor local en el talento territorial
}

\section{The social dimension of the local actor in the territorial talent}

\author{
Juan Carlos Delgado Barrios*
}

\section{RESUMEN}

Esta investigación parte de la definición del actor local en los planteamientos expuestos por García (2007), Fernández (2008) y Arocena (2002). Los actores locales con su capacidad asociativa ligada a decisiones y acciones, a influencias de poder o de experticia para el desarrollo territorial. Este artículo estudia la dimensión social, - el capital social -, del actor local, dentro del talento territorial. Esta dimensión está vinculada con la exigencia a los actores locales involucrados en el desarrollo territorial de contar con altos niveles de organización social para la producción, participación social protagónica, auto dependencia, confianza en los sistemas e instituciones de la participación, capacidad de articulación con otros actores organizados o en comunidades, así como, con otros territorios y con redes territoriales del desarrollo. Metodológicamente es una investigación exploratoria, de análisis de un territorio como laboratorio vivencial a partir de un enfoque sistémico procesal, desde la metodología sistémica interpretativa, sustentada en referencias bibliográficas y electrónicas dando apoyo a las reflexiones y experiencias del autor sobre el tema abordado de manera inductiva y constructivista. Como resultado se presentan la conceptualización de la dimensión social del actor local y su rol en el desarrollo territorial a partir de un territorio referencial bajo investigación (Zona Sur del Lago de Maracaibo. Concluyendo con laidentificación de tres escenarios de actuación del actor local dentro dela dimensión social del talento territorial.

Palabras Clave: Desarrollo Territorial, Actor Local, Talento Territorial, Capital Social, Territorios que Aprenden.

RECIBIDO: Julio 2017 ACEPTADO: Octubre 2017

\footnotetext{
* Profesor Titular Jubilado de la Universidad de Los Andes-Venezuela, Doctor en Ciencias Humanas (LUZ) y Doctor en Ciencias Gerenciales (URBE). Este artículo es parte de la investigación financiada por el CDCHTAULA bajo el código NURR-H-598-17-09-B. Mérida, Venezuela. Correo electrónico: juancdelgadoula@hotmail.com
} 


\section{ABSTRACT}

This research starts from the definition of the local actor in the approaches presented by García (2007), Fernández (2008) and Arocena (2002). Local actors with their associative capacity linked to decisions and actions, influences of power or expertise for territorial development. This article studies the social dimension, the social capital, of the local actor, within the territorial talent. This dimension is linked to the requirement to local actors involved in territorial development to have high levels of social organization for production, protagonist social participation, self-dependence, trust in the systems and institutions of participation, ability to articulate with others organized actors or in communities, as well as with other territories and with territorial networks of development. Methodologically, it is an exploratory investigation, analyzing a territory as a living laboratory based on a systemic approach, from the interpretive systemic methodology, supported by bibliographical and electronic references, giving support to the author's reflections and experiences on the topic addressed in an inductive way and constructivist. As a result, the conceptualization of the social dimension of the local actor and its role in territorial development from a referential territory under investigation (Southern Zone of Lake Maracaibo) is presented, concluding with the identification of three scenarios of action by the local actor within the social dimension of territorial talent.

Key words: Territorial Development, Local Actor, Territorial talent, Social Capital, territories learning.

\section{Introducción}

La Agenda 2030 para el Desarrollo Sostenible (CEPAL-ONU, 2017a) y Horizonte 2030 (CEPAL-ONU, 2017b) expresan un consenso sobre la necesidad de avanzar hacia sociedades más inclusivas, solidarias y cohesionadas, poniendo a las personas en el centro, promoviendo un modelo de desarrollo sostenible, haciendo un llamado a "que nadie se quede atrás" en la senda del desarrollo.

Entonces, esta propuesta trata de superar las desigualdades económicas, sociales, políticas y culturales, características de la región, desatando con estrategias factibles los nudos críticos del desarrollo que impiden a éste ser eficiente, inclusivo y equitativo. En tal orientación, proponen un esfuerzo de todos para superar el rezago social y económico a grandes sectores de la población en la región. 
En este sentido, es pertinente comprender las desigualdades a superar, evidenciadas en el documento de la CEPAL-ONU titulado La Matriz de Desigualdad Social en América Latina en él cual afirman:

.....para analizar las desigualdades sociales es necesario identificar no sólo a quienes se les ha vulnerado el acceso a este nivel básico de bienestar, sino también prestar atención a la distribución de activos, medios y oportunidades, ingresos y otros resultados, poder e influencia. En este sentido, la desigualdad hace referencia a las asimetrías en la capacidad de apropiación de los recursos y activos productivos (ingresos, bienes, servicios, entre otros) que constituyen o generan bienestar entre distintos grupos sociales. En definitiva, apunta a la concentración de una gran proporción de la riqueza en un sector reducido de la población. A su vez, refiere a la exclusión y la inequitativa distribución del poder político, que deja para unos pocos el espacio de las decisiones que afectan a una mayoría, posibilitan o limitan el ejercicio de derechos y el desarrollo de capacidades. Así, es importante resaltar que la desigualdad tiene un carácter fundamentalmente relacional y, también, que es un fenómeno indisoluble de las relaciones de poder a nivel individual y colectivo (2017 c: 18).

Desde esta perspectiva, el documento citado refuerza lo expuesto por la Organización de Estado Americanos en su informe Desigualdad e Inclusión en las Américas (OEA, 2011) donde se sugiere que las desigualdades se profundizan cuando hay carencias de poder y organización social en las sociedades locales y exige atender la inclusión social y el empoderamiento de las personas en los territorios.

Recomendando a las personas convertidos en actores locales a acudir a los procesos del desarrollo con capital social y político, con sus intereses y motivaciones, con sus visiones estratégicas involucrándose más allá de ser beneficiarios subsidiarios y pasivos de las transformaciones del territorio.

En el documento de la UNESCO-ONU (2016) denominado El Informe Mundial sobre Ciencias Sociales evidencia el crecimiento de las desigualdades y su impacto en el desarrollo. En este documento destacando 7 desigualdades, las cuales son definidas de la siguiente manera: 
La desigualdad económica: diferencias entre niveles de ingresos, bienes, patrimonio y capital, estándares de vida y empleo. La desigualdad social: diferencias entre el estatus social de distintos grupos de población y desequilibrios en el funcionamiento de los sistemas de educación, salud, justicia y protección social. La desigualdad cultural: discriminaciones basadas en el género, la etnia y la raza, la religión, la discapacidad y otras identidades grupales. La desigualdad política: capacidad diferenciada de los individuos y grupos para influir en los procesos de elaboración de decisiones en materia de políticas, así como para beneficiarse de esas decisiones y tomar parte en la acción política. La desigualdad territorial: disparidades territoriales y regionales entre núcleos centrales y periferias, entre zonas urbanas y rurales, y entre regiones con mayor o menor diversidad de recursos. La desigualdad cognitiva: disparidad en el acceso a los recursos naturales y los beneficios de su explotación; grado de exposición a contaminaciones y riesgos; y diferencias en los medios necesarios para adaptarse a esos riesgos. (2016: 5).

Estas desigualdades llevan implícito las carencias o bajos niveles de organización económica, social y política de la sociedad de esos territorios. O sea, el bajo nivel del capital social. En consecuencia, la matriz de desigualdad social en América Latina infiere la importancia de resaltar en la desigualdad su carácter fundamentalmente relacional, con énfasis en las relaciones de poder a nivel individual y colectivo. Haciendo énfasis en las interrelaciones de las personas con otras personas, con organizaciones e instituciones como estratégicas para la integración en los procesos del desarrollo.

Esta crisis del capital social o relacional en algunos territorios está obstaculizando a su desarrollo, por la dificultad de convocar a los diálogos intersubjetivos en el camino a construir comunidades, a consolidar organizaciones culturales, sociales, políticas y para la producción y para el activismo propositivo, de presión o de reclamo social, económico y sociopolítico y, con ello, limitando la posibilidad de restituir el tejido social frente a la fragmentación.

Esta situación de desbalance en la organización social y política es grave cuando los procesos del desarrollo demandan de organizaciones más complejas como 


\section{Juan Carlos Delgado Barrios}

Telos Vol. 20, No. 1 (2018). 29-57.

las comunidades y las redes sociales y las instituciones públicas y privadas vinculadas con las actuaciones en función del desarrollo territorial.

Este planteamiento permite deducir que los bajos niveles relacionales del actor local derivan en carencias en su capacidad asociativa para integrarse eficientemente en los procesos de desarrollo, para formar e involucrarse en organizaciones de participación para la decisión y la acción y, que estas insuficiencias relacionales son fuente de desigualdades sociales.

Entendido, en esta investigación, el desarrollo territorial como el producto de la comunicación -acción envolvente de los pobladores asentados en un espacio geográfico definido, sugiriendo la necesaria presencia protagónica del actor local quien acude con su talento territorial.

En esta misma dirección, el territorio es concebido como una comunidad de intereses donde el nivel de influencia o de presión de los actores locales es clave para el reconocimiento e imposición de sus intereses y motivaciones respaldados en sus relatos emergentes particulares

Esta premisa, coloca al actor local como protagonista en el desarrollo y miembro de la comunidad de intereses, específicamente, en los escenarios de dialogo en la búsqueda de construir los consensos necesarios para la transformación territorial. Exigiendo a éste capacidad asociativa, de comunicación, confianza en los mecanismos y escenarios de la participación, tolerancia y reciprocidad, o sea poseedor de capital social (dimensión social).

Además, demandas de actuación frente a los procesos de fragmentación social, política y económica, que es promotora de desigualdades y de obstáculos al desarrollo, capaces de diseñar e implementar estrategias de restructuración o recomposición del capital social.

En esta publicación, se analizan estas actuaciones mediante la declaratoria del territorio como territorios de aprendizaje, definidaen tres escenarios donde se desenvuelve el actor local: en lo particular, en sus reflexiones, intereses y 


\section{La dimensión social del actor local en el talento territorial}

motivaciones sobre el desarrollo del territorio donde convive; en los espacios de diálogo (sujetos - sujetos), diálogos intersubjetivos, construyendo comunidad; y en las redes sociales de conectividad entre comunidades y territorios.

En consecuencia, el objetivo del presente artículo está centrado en el estudio de la dimensión social del talento territorial, específicamente, el capital social del actor local en el territorio, partiendo de que el desarrollo territorial exige de los actores locales un talento territorial donde es concebido la organización, participación y movilización social, junto con la confianza de los ciudadanos y las comunidades en las instituciones e instrumentos de sociabilización, como la base para lograr el desarrollo de las capacidades de involucramiento social, político, económico y cultural.

Generando, de esta manera, valor agregado en los actores locales en la acción colectiva a partir de las conexiones interpersonales y con las instituciones $\mathrm{u}$ organizaciones del territorio a los fines de la inclusión social y política de éstos en los procesos del desarrollo territorial, dándole así, inclusión, protagonismo y coherencia social y política a las actuaciones de la sociedad local en las tareas del desarrollo, traduciéndose en elevación de la productividad, la eficiencia y el compromiso en las interacciones sociedad-territorio.

Como referente empírico para la observación y la reflexión ha sido seleccionado el territorio de la Zona Sur del Lago de Maracaibo, conformado por 17 municipios ubicados entre los estados venezolanos Zulia, Trujillo y Mérida.

Esta es una zona de extraordinarias condiciones agroecológicas e hídricas para el desarrollo agro-productivo a gran escala, sin embargo, acusa grandes carencias tecnológicas, institucionales, de organización social, de empoderamiento político y de visión prospectiva convertidas en nudos críticos, en obstáculos para emprender el desarrollo territorial.

A partir de esta observación y reflexión la formulación de la hipótesis de la investigación es expresada de la manera siguiente: el bajo nivel de capital social de la 
Juan Carlos Delgado Barrios

Telos Vol. 20, No. 1 (2018). 29-57.

población de la Zona Sur del Lago de Maracaibo es un obstáculo para promover y consolidar la organización e integración de los actores locales en los procesos del desarrollo territorial.

Esta aseveración planteada para construir la hipótesis es sustentada en investigaciones (observaciones, reflexione e inferencias) del autor en el territorio antes identificado mediante estudios estadísticos y en espacios de dialogo para el diagnóstico cualitativo y validación de las mediciones cuantitativas con participación dialógica de actores locales, permitiendo establecer algunos indicadores referidos a la dimensión social de los actores locales, tales como la existencia de relaciones de confianza y solidaridad, reciprocidad y corresponsabilidad; de normas y sanciones que institucionalicen el sistema de interrelaciones; acceso a información de los miembros del grupo; acciones colectivas y de cooperación; cohesión social e inclusión y relaciones con otros grupos; capacidad del grupo de generar acciones políticas en su entorno.

En sí, evaluar los niveles de organización social para la producción, participación social protagónica, auto dependencia, confianza en los sistemas e instituciones de la participación, capacidad de articulación con otros actores organizados o en comunidades, así como con otros territorios y con redes territoriales del desarrollo.

El desarrollo de esta evaluación de campo se logro utilizando metodologías aplicadas con casos específicos y adaptadas a este caso territorial. (Patiño et al, 2012). Así mismo, apuntalando la evaluación con la aplicación de metodologías de diálogos para validar la información resultante de la encuesta, en grupos de participación en cada uno de los municipios señalados mediante la propuesta: Espacios de Diálogos para Construir Estrategias para el Desarrollo (CEPAL-ONU, 2013).

Es de advertir que los resultados y métodos estadísticos desarrollados en la investigaciónhan sido utilizados para el diseño de las bases interpretativas y de 35 


\section{La dimensión social del actor local en el talento territorial}

hipótesis de esta investigación serán expuestos en artículos posteriores en proceso de consolidación. Por tanto, es necesario aclarar que la publicación actual es parte del sustento teórico referencial de la investigación global sobre talento territorial y sus dimensiones, específicamente en este articulo la dimensión social.

Esta estrategia investigativa, expuesta en este artículo, busca, además de elevar el talento territorial (Delgado, 2015), específicamente en la dimensión social, para hacer más productivas y eficientes las actuaciones de los actores locales en el desarrollo, aportar elementos para superar las desigualdades y la exclusión social derivada de carencias asociativas de las comunidades locales.

El enfoque sugerido como metodología de análisis y de construcción de las propuestas, en esta publicación, es el enfoque sistémico-procesal desde la metodología sistémica interpretativa de Villafuerte (2008), -entendimiento y comprensión del territorio y sus actores locales-.

Este enfoque, permite, desde el pensamiento complejo (Morin, 2009; Soto González, 1999) visualizar al territorio como una complejidad conformada por sistemas en redes de comunicación-acción, de diálogos-consensos y, donde su fluidez y dinámica están marcadas por la existencia de procesos, flujos de información, de decisiones y de materiales, altamente articulados y activos bajo la presencia del factor humano quien es el activador de los procesos dentro del territorio o comunidad de intereses. En esa misma dirección, la metodología asumida en la investigación es de tipo exploratoria-documental, (Hurtado, 2000: 217).

La estructura del artículo es conformado, en una primera parte, con el análisis interpretativo de la dimensión social en el contexto del territorio referencial, destacando en esta parte, la definición del actor local como agente de desarrollo, el establecimiento del territorio como contexto de la dimensión social, para concluir, definiendo la dimensión social de actor local como dimensión del talento territorial. En una segunda parte, con el análisis de los escenarios de actuación de los actores locales local. Conclusiones y Referencias Bibliográficas. 
Juan Carlos Delgado Barrios

Telos Vol. 20, No. 1 (2018). 29-57.

\section{Análisis Interpretativo de la Dimensión Social en el contexto del territorio referencial.}

A partir de la observación e interpretación de los procesos del desarrollo territorial y el sistema de interrelaciones de los actores locales en el contexto de análisis referencial: la Zona Sur del Lago de Maracaibo - Venezuela estos son expuestos en los siguientes términos:

\section{El actor local en los procesos del desarrollo}

En esta investigación parte de la definición de actor local expuesta por Arocena (2002: 25, 26) cuando indica que el actor local es un individuo, grupo o institución cuyo sistema de acción coincide con los limites (intereses) de la sociedad local. Este autor relaciona al actor local con la escena donde se desenvuelve la acción, en donde simultáneamente es motor y expresión del desarrollo local, movido por motivaciones e intereses hacia propósitos comunes de y con otros actores locales en un escenario definido. Además, deja claro que el actor local en funciones de protagonista en el territorio es un agente del desarrollo (actor-agente). Específicamente, en el campo político, económico, social y cultural son portadores de propuestas que tienden a capitalizar mejor las potencialidades locales.

Esta definición de Arocena (2002) del actor local cumple con las exigencias de Bourdieu(1998), analizado por Fernández (2008), al advertir que el agente exhibe al individuo como un reproductor de prácticas, como un operador, mientras el concepto de actor le acrecienta al individuo los límites de su decisión y de su acción, le da mayor autonomía de opinión, vinculación y gestión, con lo que se le concibe como alguien capaz de convertirse en un creador o innovador en el ámbito de la acción.

En esta misma dirección, García (2007) complementa los planteamientos anteriores, destacando que el actor es un individuo o colectivo concurriendo a los procesos y contextos del desarrollo con sus intereses, motivaciones, percepciones y 


\section{La dimensión social del actor local en el talento territorial}

creencias; poseedor de capacidad y disposición para la participación y la organización; dispuesto a participar en la resolución de conflictos y en la construcción de consensos; capaz de construir y establecer sus criterios sobre las realidades y problemas que le ocupan, lo que le da una identidad particular a su pensamiento y acción. En otras palabras, un actor es una unidad de decisión-acción responsable.

Desde este marco definitorio del actor local, en esta parte del trabajo, la investigación trata de declarar al actor local como un individuo responsable, pensante y actuante en su contexto. Rescatando la subjetividad del actor local desde la complejidad de sus interrelaciones particulares, no sólo en la relación comunitaria/colectivista sino en su individualidad con sus intereses y motivaciones que lo inducen a incorporarse al desarrollo de manera protagónica dentro de la comunidad de intereses del territorio.

Es preciso, entonces, refundar el rol del actor local en los procesos de desarrollo, desde la complejidad de sus interrelaciones, no cayendo en la simplificación del sujeto aislado, tal como lo advierte Soto González al estudiar a Edgar Morín en Complejidad y Sujeto:

La complejidad es relación y es inclusión; ni siquiera excluye la simplificación, sino que la integra como uno de los elementos del pensamiento complejo.........para el pensamiento complejo es esencial considerar al sujeto y a todos los mecanismos que lo condicionan, o sea, conocer nuestro conocimiento o nuestras formas de conocer. Es necesario, por tanto, introducir al sujeto, lo que equivale a fundarlo sobre nuevas bases, es decir, refundarlo (1999:10).

Estas posiciones complementadas con Delgado (2015) quien aporta su visión sobre el rol del actor local en el territorio:

....el desarrollo está relacionado con las capacidades y actuaciones de transformación de los factores humanos sobre el territorio, más allá de las potencialidades culturales, ambientales, económicas, sociales y tecnológicas. En este caso, el factor humano no sólo acude con sus capacidades, competencias y habilidades, sino que se incorpora como parte de la comunidad de interesados en el desarrollo, exigiendo capacidad de 
organización, de participación, poder de influencia, creatividad, pensamiento estratégico, ética ambiental y cultural bajo la égida de la sustentabilidad y la responsabilidad social. (2017:88).

En los escenarios del desarrollo territorial concurre el actor local con su talento territorial, definido éste como el conocimiento - relacional distribuido en un territorio visto desde el sujeto, como la presencia protagónica de los actores locales en un territorio con su ética frente a lo cultural, lo ambiental, lo urbano y con la convivencia humana, con sus intereses y motivaciones, sus visiones del desarrollo, su capacidad asociativa, su liderazgo, prospectiva, su poder de influencia, sus competencias y habilidades para la transformación de las realidades territoriales.

En marco definitorio realza la subjetividad del actor local desde el pensamiento complejo asumiendo el análisis de Pozzoli quien indica:

El sujeto se introduce progresivamente a la realidad en un proceso dialógico y reflexivo, que implica la expansión y reorganización de la conciencia. Conciencia inicialmente sujetada por el control social funcionalista. A partir del ejercicio de la reflexividad, el sujeto comienza a auto-observarse, a desarrollar la auto-critica, a interpretarse en el ejercicio de su capacidad de sujeto histórico, lo que le permite recuperar su protagonismo y orientarse de un modo más autónomo. El sujeto al asumir su complejidad, re-descubre otra definición de si mismo y desde aquí una manera diferente de relacionarse con su entorno (2006: 5).

Esta perspectiva busca analizar las actuaciones del actor local en el desarrollo territorial quien reflexiona sobre sí mismo y su relación con el entorno. En consecuencia, para estudiar el rol del actor local en el desarrollo territorial es pertinente conceptualizar el desarrollo territorial como la comunicación-acción envolvente de la sociedad local en su relación con el espacio geográfico donde cohabitan, donde las personas conviven en la localidad involucrándose ecológica y éticamente para la transformación del territorio.

En esta enunciación, la presencia de las personas y su pensamiento- acción particular como principal factor del desarrollo es destacada (Habermas, 2003), no sólo 


\section{La dimensión social del actor local en el talento territorial}

como fuerza hacedora, sino además, por su capacidad de convivencia en armonía, tanto con sus valores de la trascendencia, con la cultura, la naturaleza y las otras personas, así mismo, por su capacidad interpretativa de sus realidades a los fines de transformarlas, por su participación y organización, por su capacidad de influencia y disposición para consensuar y manejar conflictos en la búsqueda del desarrollo territorial, por su ética de la sustentabilidad, por su sentido de la calidad de los procesos que desarrollan y por su talento humano y capacidades de transformación (competencias y habilidades).

De allí, el desarrollo territorial constituye una nueva forma de mirar y de actuar en un territorio donde se involucran los distintos actores que conviven en la localidad, convirtiendo la participación social protagónica en un factor clave en el propósito de diagnosticar juntos, construir visiones compartidas, diseñar propuestas de transformación e implantar estrategias con su consecuente autoevaluación.

Tratándose entonces, con el desarrollo territorial, de activar e involucrar a la sociedad local en los procesos de desarrollo; esto es: el actor local deja de ser un agente solitario y pasivo para convertirse en un sujeto interconectado, reconocido en sus relatos particulares y comunitarios, en consecuencia, es necesario la voluntad, disposición, la asociatividad, la participación organizada y la confianza y resonancia de los actores locales para elevar la eficiencia de la ecuación humana con relación a la transformación territorial.

En estos escenarios, el actor local promociona y desarrolla cualidades proactivas para implicarse, así como, de conectividad y empoderamiento para liderar y para la coordinación en la búsqueda de promover y gestionar el poder colectivo de transformación y, de comunicación-acción de los actores locales a objeto de influir, concretar consensos y manejar conflictos a los fines del diseño, implantación y evaluación de estrategias de desarrollo; lo cual le demanda aptitudes y voluntad para la participación en el sistema de decisiones donde se involucra.

Este planteamiento es apoyado en Buciega y Esparcia cuando exponen: 
Podríamos referirnos al capital social como los recursos que se derivan de la existencia de determinada forma de relaciones sociales. La pertenencia a unas redes permite al sujeto o al grupo acceder a unos recursos a los que, en ausencia de las mismas, no podría o le resultaría mucho más costoso; por recursos nos referimos a obligaciones de reciprocidad, normas y sanciones, acceso a información, reducción de costes de transacción, y esto a su vez puede derivar en compromiso y responsabilidad, confianza, mayor democracia, innovación, acción colectiva (2013: 35).

Más adelante estos autores señalan:

“...hay que prestar atención al potencial del capital social como instrumento y no tanto como fin en sí mismo, y en este sentido puede ayudarnos a explicar mejor las dinámicas de desarrollo territorial; con relación a ello hay que tener en cuenta dos aspectos fundamentales, el potencial del capital social para generar acción colectiva, y la importancia del marco institucional, que pueden reforzar o para debilitar el capital social y su desarrollo(2013: 87).

Esta actuación del actor local desde el sujeto en el desarrollo territorial es lo que configura la dimensión social del actor local dentro de las dimensiones del talento territorial. Delgado (2015).

\section{Territorio como contexto.}

Los emergentes escenarios derivados de los procesos de descentralización del Estado y las expectativas del desarrollo territorial exigen espacios de participación ciudadana con organización social y política de la población, miradas prospectivas o de pensamiento estratégico, actuaciones éticas con la sustentabilidad cultural, ambiental y la convivencia humana, indudablemente demandan cambios significativos en el pensamiento y la acción de los actores locales con respecto a su protagonismo en el territorio.

Los actores locales involucrado en el desarrollo se desenvuelven en un territorio definido más allá del espacio geográfico y su patrimonio ambiental, cultural, económico y tecnológico, para concretarse como una comunidad de intereses donde 


\section{La dimensión social del actor local en el talento territorial}

acuden a encuentros de diálogos para mediar, concertar, imponer y negociar sus perspectivas de las transformaciones a mediano y largo plazo del territorio en cuestión.

Este planteamiento es reforzado con Alburquerque y Pérez quienes afirman:

El territorio, objeto de la transformación, es el conjunto de actores ya gentes que lo habitan, con su organización social y política, su cultura e instituciones, así como el medio físico o medioambiente del mismo. Se trata de un sujeto (o "actor") fundamental del desarrollo, al incorporar las distintas dimensiones de éste, es decir, el desarrollo institucional, cultural y político, el desarrollo económico, el desarrollo sustentable ambientalmente, y el desarrollo social y humano (2013: 2).

Los autores antes citados reconocen la presencia del actor local como activador fundamental del desarrollo territorial, significando las interrelaciones entre sujetos (actores y agentes) como fuentes de formación y actuación de instituciones y organizaciones en el territorio.

A su vez, Vázquez-Barquero expone en cuanto a la relación territorio y capital social, lo siguiente:

El territorio puede entenderse, por lo tanto, como el entramado de intereses de todo tipo de una comunidad territorial, lo que permite percibirlo como un factor de desarrollo, siempre que sea posible mantener y desarrollar la integridad y los intereses territoriales en los procesos de crecimiento y cambio estructural.... Los actores locales, a través de sus iniciativas y decisiones de inversión y de la participación en la formulación y gestión de las políticas, contribuyen al desarrollo y la dinámica productiva de una localidad, un país o un territorio (2007:188).

A partir de estas consideraciones definen al territorio como una comunidad de intereses, de sujetos interesados en el desarrollo territorial, donde acuden con sus motivaciones, intereses, interpretaciones de la realidad, visiones prospectivas y su voluntad y capacidad para la asociatividad y el protagonismo social.

La presencia del actor locales re-direccionada y complementada al encontrarse e integrarse con otros (sujeto-sujetos) en espacios de diálogo y de redes de 


\section{Juan Carlos Delgado Barrios}

Telos Vol. 20, No. 1 (2018). 29-57.

comunicación-acción para dirimir conflictos y construir consensos sobre las transformaciones de la realidad territorial construyendo la comunidad de intereses.

Como comunidad de intereses, el territorio es una comunidad política donde el poder de influencia o de presión es clave en la construcción de las visiones compartidas del desarrollo territorial, pero previo a la capacidad de influencia es indispensable, como pre requisito, la existencia de confianza en los escenarios e instrumentos de la participación y de la construcción de los diálogos intersubjetivos, por tanto, constituyen estos elementos fortalezas para la conectividad, organización y movilización social; esto quiere decir, capital social de los actores locales involucrado en el desarrollo territorial.

Estos supuestos desarrollan la estrategia de análisis de la dimensión social del talento territorial al sugerir la contextualización en su escenario de actuación, que es el territorio. En primer lugar, el actor local, desde lo particular, reflexiona sobre sus intereses y motivaciones en el desarrollo del territorio, auto reconociéndose como un protagonista de la comunidad de intereses, creando su propio relato de la realidad territorial.

En segundo lugar, convoca o responde a la convocatoria a dialogar con otros actores locales, diálogos intersubjetivos, para intercambiar sobre los relatos de la realidad territorial y sus perspectivas de desarrollo. Iniciándose así la construcción de la comunidad de intereses, de la construcción del relato interpretativo de la comunidad, de los encuentros de los intereses comunes, de los consensos o la resolución de los conflictos, de las perspectivas o intereses.

En tercer lugar, reconoce el territorio donde desarrolla su acción social como una conformación de comunidades o sectores interconectados en redes de comunicación-acción para construir y gestionar la visión compartida hacia el plan integral del territorio. Significando que el actor local asociad y empoderado asume una visión prospectiva de la realidad territorial, en consecuencia, haciéndose protagonista de los procesos globales del desarrollo territorial. 


\section{La dimensión social del actor local en el talento territorial}

El desarrollo de la conceptualización y caracterización de la dimensión social del actor local, parte de identificar el sistema de interrelaciones que el actor local sostiene en un territorio y dentro de éstas, su pensamiento-acción.

Dematteis y Governa en esta dirección sostienen.

Los actores locales desarrollan en el interior del territorio relaciones de tipo cooperativo, de creación de consensos, competitivo y de conflicto, a través de las cuales se hace posible una visión estratégica y una acción colectiva orientada hacia objetivos compartidos de desarrollo (2005: 48).

Infiriendo que la sociabilización del actor local expresada en la confianza hacia los escenarios y mecanismos de la participación, la reciprocidad, la otredad, la capacidad de convocar o ser convocado para establecer diálogos intersubjetivos a los fines de resolver conflictos o construir consensos es clave en el desarrollo territorial. A esta sociabilización del actor local es denominada capital social y representa la dimensión social como dimensión del talento territorial, vista desde el sujeto en la complejidad de su pensamiento-acción.

En esta misma dirección, Delgado (2017)en su intento de rescatar la subjetividad del actor local en los procesos del desarrollo, define el talento territorial como el conocimiento, aptitudes, intereses y motivaciones distribuidos un territorio visto desde el sujeto, significa la presencia protagónica de los actores locales en un territorio con su ética frente a lo cultural, lo ambiental, lo urbano y en la convivencia humana, con sus intereses y motivaciones, sus visiones del desarrollo, su capacidad asociativa, con su liderazgo, con su poder de influencia, con sus competencias y habilidades para la transformación de las realidades territoriales.

Se infiere, entonces, el talento territorial está definido en el marco de su complejidad por múltiples factores identificados con el rol de los actores locales en los procesos del desarrollo territorial, estos factores conforman dimensiones, a saber: 
Juan Carlos Delgado Barrios

Telos Vol. 20, No. 1 (2018). 29-57.

La dimensión interpretativa de la realidad territorial: donde el desarrollo territorial exige de los actores locales tener un talento territorial con disposición de encontrarse e interpretarse consigo mismo, con sus pares y con la realidad donde convive mediante procesos de comunicación-acción en redes intersubjetivas, desarrollando la capacidad interpretativa de la realidad del territorio donde está inmerso con sus acciones y procesos de vida. Significa el desarrollo de espacios de entendimiento mediante los diálogos intersubjetivos de argumentación contextualizada donde el lenguaje (conocimiento) como acción comunicativa sustenta y facilita los consensos de los actores locales interesados en el desarrollo territorial.

La dimensión prospectiva estratégica: significa el pensamiento futurista del sujeto involucrado en el desarrollo territorial, esta dimensión plantea la necesaria mirada a lo lejos, son los mapas mentales anticipándose a una realidad por hacer y construir, anticipándose y preparándose a tiempo, para proyectar el futuro, para identificar los nudos críticos del desarrollo, la capacidad prospectiva para el diagnóstico estratégico y el diseño y selección de las líneas estratégicas del desarrollo territorial. Es pensar el territorio desde la complejidad con una cultura hacia el horizonte, hacia el deber ser, en escenarios tendenciales sobre el futuro.

La dimensión del capital social: el desarrollo territorial exige a los actores locales un talento territorial con altos niveles de organización social para la producción, participación social protagónica, auto-dependencia, confianza en los sistemas e instituciones de la participación, capacidad de articulación con otros actores organizados o comunidades, así como, otros territorios y con redes territoriales de desarrollo.

La dimensión política (del poder): el desarrollo territorial exige de los actores locales un talento territorial relacionado con el empoderamiento ciudadano y comunitario, entendido éste, como la capacidad de liderar y de coordinar para promover y gestionar el poder y la comunicación de los actores locales a los fines de construir consensos y manejar conflictos en la estructuración, implantación y 45 


\section{La dimensión social del actor local en el talento territorial}

evaluación de estrategias del desarrollo, así como, de aptitudes y voluntad para la participación en el sistema de decisiones donde está involucrado.

La dimensión de la ética y la sustentabilidad territorial: el desarrollo territorial exige de los actores locales tener un talento territorial comprometido éticamente con la sustentabilidad cultural, ambiental y urbana del territorio, donde desarrolle sistemas de producción con responsabilidad social y ambiental bajo el paradigma de la economía solidaria; sustentado en un pensamiento ecológico y de articulación respetuosa con los diversos patrimonios territoriales.

La dimensión de la calidad territorial: el desarrollo territorial exige de los actores locales un talento territorial comprometido con la calidad ambiental, urbana, institucional y de los productos derivados de los procesos culturales, sociales, tecnológicos, económicos y administrativos producidos en el territorio. A su vez, comprometido con la transparencia administrativa y la entrega de cuentas, tanto en el sector público, como en el sector privado.

La dimensión de las capacidades de transformación: el desarrollo territorial demanda de los actores locales un talento territorial con capacidades motivacionales, espirituales, intelectuales, creativas, de emprendimiento y tecnológicas para la transformación de las realidades del contexto donde se desenvuelve en la búsqueda del logro del desarrollo territorial. Las siete dimensiones integradas de manera transversal conforman el talento territorial, teniendo como eje central la dimensión social, es decir, el actor local actuado, comunicándose, participando e integrándose en comunidades y en redes de comunidades o territorios.

La dimensión social desde la perspectiva del talento territorial, parte de la participación del actor local en la comunidad de intereses que configura el territorio y luego con su pensamiento y acción desplegado en los escenarios y procesos del desarrollo territorial donde la voluntad y la confianza en la participación y sus mecanismos junto con la reciprocidad y el activismo social, son claves para definir esta dimensión. 
Juan Carlos Delgado Barrios

Telos Vol. 20, No. 1 (2018). 29-57.

\section{Escenarios de actuación del actor local.}

Los escenarios de la dimensión social del actor local están definidos por las actuaciones de éste como individuo, en sus relaciones con otros formando comunidad y, en los procesos de formación de redes sociales. Estos son:

\section{Escenario desde el sujeto}

En este escenarioconcurre el actor local en su individualidad en un ejercicio de la reflexividad, desencadenando un proceso de auto-observación, de auto-critica, de desarrollo de su capacidades interpretativa, prospectiva y de la sustentabilidad en el ejercicio de su capacidad de sujeto histórico, reconquistando su protagonismo y orientándose de un modo más autónomo. Asume su complejidad desde él y su circunstancia, re-descubre otra definición de si mismo, replanteándose una manera diferente de relacionarse con su entorno.

Esto configura una indagación desde el sujeto y su relación con el contexto territorial a partir de sus intereses y motivaciones personales concretando en la argumentación hacia la construcción de su relato interpretativo particular. Es un acercamiento a la realidad a objeto de escudriñar la dinámica y los procesos de ésta y su relación con respecto a sus motivaciones particulares midiendo siempre su capacidad u oportunidad de influencia, de participación, de involucramiento y conectividad con otras personas e instituciones.

Es una toma de conciencia y de confianza de su rol como animador y transformador local, en términos de sus capacidades y compromisos con la transformación de la realidad territorial, comprendiendo la valorización de sí mismo como factor clave con influencia en el territorio en el marco de la ciudadanía: conocimiento de sus derechos y deberes, confianza en la participación, autoestima personal sobre el valor de su interés, reconocimiento de la presencia de otros actores para compartir visiones, disposición a comunicarse y actuar en los procesos del desarrollo territorial. Es lo que Gardner denomina en su obra Inteligencias Múltiples (2011) el desarrollo de la inteligencia intrapersonal. 


\section{La dimensión social del actor local en el talento territorial}

La dimensión social particular del actor local es clave para acrecentar su dimensión política (poder de influencia y presión): confianza en los espacios y mecanismos de participación, voluntad para convocar y ser convocado, sentido de responsabilidad, reciprocidad, solidaridad, resonancia y otredad, dentro de estas aptitudes su capacidad de comunicar su relato particular juega un papel significativo a la hora de acrecentar los niveles de empoderamiento y conectividad social, así como, superar las condiciones de exclusión.

Siendo el talento territorial una composición compleja, las estrategias de aprendizaje, en este escenario, deben ser desarrolladas atendiendo las especificidades particulares de los actores locales dentro de la globalidad de la comunidad de intereses del territorio donde como contexto. Aprendizaje a desarrollarse de manera integrada /transversal a las otras dimensiones del talento territorial.

Desde la escuela básica hasta el nivel universitario se deben desplegar estrategias de Investigación-Acción-Participativa (Villasante y otros, 2000) y ,el desarrollo de escenarios y prácticas de activismo cultural, social y político para acrecentar la dimensión social de la población en general. El activismo cultural, social y político en los territorios es un escenario muy importante para el aprendizaje e innovación de formas y estrategias de participación social en la dirección de acrecentar la dimensión social particular del actor local.

\subsection{Escenario desde los espacios de diálogo:}

El proceso de análisis de este escenario apoyado por lo expuesto por Habermas (2003) y la investigación sobre Habermas efectuada por Díaz Montiel

El diálogo intersubjetivo como proceso racional, puede hacer posible la construcción de nuevos mecanismos sociales que configuren los fines más justos, a través de mecanismos colectivamente consensuados, mediante ejercicios de argumentación, que conlleven a la construcción de un nuevo proyecto de vida social. Los preceptos moral-discursivos que funden dicho proceso, estarán dados por la propia fuerza del sentido intersubjetivo que da la capacidad de abrirse a la consideración de los fines de otros, para lograr la integración social construida desde la voluntad general (2007:9). 
Este nivel es planteado desde una nueva forma de comunicación, la comunicación dialógica en el territorio, es decir, generando espacios de intercambio signados por la confianza; el compartir de conocimientos, informaciones, capacidades y recursos; por la construcción de consensos y visiones compartidas; por interrelaciones horizontales. Resulta entonces, una comunidad de comunicación sujeta a las necesidades de cooperación y solidaridad humana.

Los espacios de diálogo constituyen una fase de construcción de comunidad en la relación sujeto-sujetos en la comunidad de intereses definitorios del territorio, este encuentro, además de democrático, debe ser dialógico, horizontal, abierto, sin aprehensiones y desde la otredad.

Para el logro de esta fase es necesaria la capacidad de convocatoria, de asociatividad, de integración de visiones en consenso, de relatoría en la dirección de recoger los relatos emergentes, de tolerancia hacia la diversidad de opiniones y propuestas. Es un esfuerzo en la dirección de la construcción comunitaria del futuro territorial desde una visión compartida.

Este nivel tiene relación con el encuentro de los actores locales. Éstos acuden a los espacios de diálogo intersubjetivo con su empatía, con su capacidad para comprender a los demás, con su tolerancia, con claridad en sus opiniones o posiciones a los fines de intercambiar sus verdades y perspectivas particulares sobre el desarrollo territorial con respecto al futuro del territorio y la responsabilidad de los actores locales en los procesos del desarrollo territorial.

En este escenario los actores locales involucrados en un proceso de irradiación y al mismo tiempo, de convocatoria de la diversidad de visiones derivadas de la multiplicidad de intereses definen al territorio a transformar a los fines de construir los consensos necesarios y posibles, así como, para dirimir y superar conflictos de intereses pudieran plantearse en torno al desarrollo territorial.

En el proceso dialógico es clave el capital social grupal y/o institucional, asociado con el actor local y la capacidad interpretativa de la realidad para concretar 49 


\section{La dimensión social del actor local en el talento territorial}

sus aportes en el encuentro sujetos-sujetos. La capacidad de influencia en los espacios de diálogo es directamente proporcional a los niveles asociativos de cada participante o interesado, -grupos de intereses- para imponer o negociar sus propuestas de transformación.

Para ello, es necesario alcanzar ciertos rangos de organización y de aprendizaje en la participación social, de canales de participación y de cultura asociativa poniéndose a prueba la capacidad de influencia, o sea, es decir, su capacidad comunicativa-convencimiento, de negociación, de construcción de consenso o manejo de conflictos aprendida en el primer escenario. Gardner (2011) lo denomina el desarrollo de la inteligencia interpersonal.

A su vez, la CEPAL-ONU (2013: 7) califica a los espacios de diálogo entre actores clave de la sociedad para construir estrategias de desarrollo público-privadas como importantes y necesarios para desarrollar iniciativas integrales y sostenibles. Destacando que, en la actualidad, las estructuras sociales y económicas están tan relacionadas entre sí que las acciones de unos actores repercuten en las de otros.

Esta perspectiva, sugiere que el dialogo sea interpretado como una herramienta universal usada y probada por las sociedades del mundo antiguo y ahora con más intensidad y pertinencia en la contemporaneidad para superar los conflictos y construir consensos, específicamente para superar la fragmentación social y política. Los espacios de diálogo entre múltiples actores son un instrumento vigoroso para promover, formular e implementar políticas públicas.

Los espacios colaborativos (generalmente conocidos como mesas de trabajo) van un poco más allá, ya que, gracias a su carácter más permanente y de integración de factores interesados, pueden llegar hasta la implementación de acciones, una vez institucionalizadas son convertidos en fortalezas propositivas en el desarrollo territorial.

El aprendizaje de la dimensión social del talento territorial, en este nivel, constituye un proceso de cambio de las representaciones sociales (Jodelet,1986) 


\section{Juan Carlos Delgado Barrios}

Telos Vol. 20, No. 1 (2018). 29-57.

individuales y colectivas en la relación del actor local con otros actores y con las instituciones relacionadas con la participación social, política y económica; es decir, implica un proceso de cambio de los pensamientos en la manera de asociarse, en las formas de influencia, del manejo de conflictos, en la construcción de los consensos, de la participación dialógica, de confianza hacia los escenarios y mecanismos de participación política y social y de auto dependencia o dependencia frente a los núcleos de poder o de acción social de la sociedad. En sí, constituye una reingeniería de pensamiento, tal como lo recomienda Morín (2002) en su obra "Los 7 saberes necesarios del futuro".

\section{Escenario desde las Redes de Comunicación-Acción.}

Esta investigación considera al territorio como un conjunto de comunidades o sectores donde cada comunidad o sector constituye un espacio de diálogo intersubjetivo unidos en intereses comunes en la globalidad territorial. A estas unidades geográficas o de intereses son denominadas los nodos y, su interrelación, red de comunicación-acción del territorio.

El territorio, desde esta perspectiva, es un sistema de interrelaciones formales e informales activadas a los fines de comunicar, dialogar y consensuar los intereses concurrentes desde la diversidad de los actores locales, donde los fines, la intensidad y la institucionalidad de esta conectividad en red define su carácter específico. Los fines de esta red de comunicación-acción territorial es concertar e integrar la visión global del territorio, es decir, la visión consensuada del desarrollo territorial.

Las redes de la dimensión social en el talento territorial integran, no sólo a los sujetos como actores locales protagónicos sino, además, acuden a ésta, las voces y motivaciones de las comunidades organizadas derivadas de los diálogos intersubjetivos sectoriales interconectados a través de una malla invisible conformada por nodos y conectores (información y decisión).

Según Delgado 


\section{La dimensión social del actor local en el talento territorial}

La noción de redes (sociales, políticas y económicas) es inseparable de la noción de relaciones, las redes son un conjunto de relaciones que toman formas imaginarias de red, estableciéndose compromisos entre los actores involucrados en la red. Las redes no responden a un plan preconcebido sino a su lógica determinada a medida que se va tejiendo. Esto quiere decir que la red es producto de una visión compartida de los tejedores donde cada uno expone su visión particular y su interés y ésta no es independiente de sus miembros (2003: 33).

La dimensión social desde el pensamiento de las redes exige una nueva postura intelectual, de pensamiento y acción del actor local, quien debe ampliar su visión hacia la complejidad que este modelo emergente expresa. Las redes en la dimensión social permiten encuentros transversales y descubrimientos de nuevos actores sociales en otras comunidades o territorios con posibilidades y aspiraciones de ser reconocidos en sus intereses particulares y colectivos como parte de un tejido invisible estableciendo un novedoso protagonismo social.

En los territorios de aprendizaje es recomendable diseñar y poner en marcha estrategias de aprendizaje del pensamiento reedificado, metodologías de construcción y gestión de redes comunitarias para el desarrollo territorial, con énfasis en la gestión de la dimensión social desde esta emergente manera horizontal y transversal de ver la organización y el poder comunitario.

Este escenario de aprendizaje del actor local, en el marco de las teorías de las redes, es muy pertinente porque las redes sociales son instrumentos de reposición o reconstrucción del tejido social, sobre todo, en la crisis del Estado centralista, de los sistemas de partidos, de los gremios y sindicatos, entre otras formas asociativas de la sociedad intermedia en las relaciones del Estado y la sociedad.

Las redes como pensamiento reedificado exigen una mirada holística del territorio, una visión desde la complejidad, derivada ésta de la diversidad de dimensiones y factores interconectados. En este escenario el actor local debe romper con su mirar reduccionista o unidimensional para abrir su pensamiento a la complejidad, a la transversalidad relacional. Esto configura un cambio o amplitud de 
las representaciones sociales de la sociedad y su protagonismo en el desarrollo territorial.

En estos tres niveles acude el actor local con su talento territorial integrando de manera transversal la dimensión social con las otras dimensiones del talento territorial, vale decir, capital social, capacidad prospectiva, capacidad interpretativa, ética de la sustentabilidad, sentido de la calidad y capital humano

\section{Conclusiones.}

Conceptualizar la dimensión social dentro del talento territorial exige, en una primera fase, interpretar al actor local desde su inteligencia intrapersonal definiendo su condición particular y sus motivaciones ante la posibilidad de interconectarse con otros actores. En una segunda, relacionar sus particularidades personales con la inteligencia interpersonal del sujeto, capacidades para las interrelaciones, que definen la eficiencia para interactuar en los espacios de diálogo sujeto-sujetos mediante la empatía como aptitud-fuerza para lograr la conexión interpersonal y, en la tercera fase ,incorporar al análisis la conectividad intercomunidades o inter territorios mediante las redes sociales.

Las carencias de capital social (dimensión social) particulares del actor local introducen factores negativos en la conformación de las comunidades de los territorios en desarrollo, debilidad que deriva en exclusión o autoexclusión de grandes sectores de la sociedad local, profundizando los niveles de desigualdad.

Cuando esta carencia es permanente y masiva acrecienta la fragmentación social, política y social, convirtiéndose este fenómeno en rémora pesada para el desarrollo, sobre todo, el desarrollo territorial que exige del protagonismo social y político de los pobladores asentados en el territorio.

Así mismo, la conformación de espacios de diálogos son clave para lograr elevar el capital social territorial en cuanto a la elevación de las fortalezas comunitarias para resolver conflictos, construir consensos y visiones compartidas, así 
como acuerdos comunitarios para la implantación, gestión y evaluación de los planes de desarrollo territorial.

Las redes como organizaciones emergentes frente a los procesos de fragmentación derivadas de la globalización y de la misma crisis del Estado centralista ocupan un lugar muy significativo en la definición y consolidación de la dimensión social del actor local, exigiendo desplegar esfuerzos hacia el desarrollo del pensamiento reedificado, de metodologías de construcción y administración de redes sociales-territoriales vinculadas con los procesos del desarrollo territorial.

Desde esta mirada, el aprendizaje de la dimensión social es estratégico a la hora de emprender el desarrollo territorial ya que la activación de la sociedad local de manera eficiente y eficaz depende de la capacidad asociativa, de la voluntad de participar, de la confianza, del interés y motivación de los actores locales en los procesos de transformación territorial.

De igual forma, es justo declarar a todo el territorio en desarrollo como un escenario de aprendizaje, o sea un territorio de aprendizaje continuo y permanente. El aprendizaje del talento territorial, específicamente la dimensión social, debe ser envolvente, incluyente y permanente entendiendo, los procesos de desarrollo como procesos continuos en el tiempo.

\section{Referencias Bibliográficas}

Arocena, José (2002). EI Desarrollo Local: Un desafío contemporáneo. Segunda Edición, Editorial Taurus -Universidad Católica. Uruguay

Alburquerque, Francisco y Pérez, Sergio (2013). Desarrollo Territorial: Enfoques,

Contenido y Políticas. Programa Enfoque sobre el Desarrollo Territorial: Banco Interamericano de Desarrollo. EE.UU.

Bourdieu, Pierre (1988), Espacio social y poder simbólico, Cosas Dichas. Editorial Gedisa Argentina.

Buciega, Almudena y Esparcia, Javier (2013). Desarrollo, Territorio y Capital Social. Un análisis a partir de dinámicas relacionales en el desarrollo rural. En: REDES- Revista hispana para el análisis de redes sociales. Vol.24, $\mathrm{N}^{\mathrm{o}} 1$, Junio 2013. Universidad Autónoma de Barcelona. España. (Pp. 81-113). 
CEPAL-ONU (2013). Espacios de diálogo para construir estrategias de desarrollo público-privadas: una guía metodológica.Publicaciones CEPAL-ONU. Chile. Extraído de http://repositorio.cepal.org/bitstream/handle/11362/27169/1/M20130025 es. pdf consulta: 20/12/2014.

CEPAL-ONU (2017 a). Agenda 2030 y los Objetivos de Desarrollo Sostenible: Una oportunidad para América Latina y el Caribe. Publicaciones CEPAL-ONU. Chile. Extraído de http://repositorio.cepal.org/bitstream/handle/11362/40155/10/S1700334_es.p df consulta: 25/10/2016.

CEPAL-ONU (2017 b). Horizonte 2030: La igualdad en el centro del desarrollo sostenible. Publicaciones CEPAL-ONU. Chile. Extraído dehttp://repositorio.cepal.org/bitstream/handle/11362/40159/4/S1600653_es. pdf consulta: 20/07/2017.

CEPAL-ONU (2017 c). La matriz de la desigualdad social en América Latina. Publicaciones CEPAL-ONU. Chile. Extraído de http://repositorio.cepal.org/bitstream/handle/11362/40668/4/S1600946_es.pd f consulta: 12/07/2017.

Crespo, Luis (2006). Espacio, territorialidad y poder. En: Revista Ciudades, Revista Trimestral de la Red de Investigación Urbana. N ${ }^{\circ}$ 70, Abril-Junio 2006, México. (Pp. 17-22).

Delgado, Juan (2003). Estrategia Metodológica para la construcción de Redes Comunitarias Agrícolas en pro del Desarrollo Local: Caso: Productores de Plátano del Sur del Lago de Maracaibo-Venezuela. En: Revista Agroalimentaria $^{\circ}$ 17. Julio-Diciembre. Universidad de Los AndesCDCHTA, Venezuela. (Pp. 29-38).

Delgado, Juan (2015). Talento Territorial: Conceptualización y Dimensiones. En: Revista AGORA -Trujillo. Año $18 \mathrm{~N}^{\circ} 36$ Julio-Diciembre. Universidad de Los Andes - CDCHTA, Venezuela. (Pp. 119-138).Extraído de http://www.saber.ula.ve/bitstream/123456789/42523/3/articulo6c.pdf consulta: 23/06/2016.

Delgado, Juan (2017).Gestión del talento territorial para el desarrollo: Territorios que aprenden. En: Revista Visión Gerencial. Año 16, $\mathrm{N}^{\mathrm{o}}$ 1, Enero Junio. Universidad de Los Andes -CDCHTA,Venezuela. (Pp. 87-102).Extraído de http://erevistas.saber.ula.ve/index.php/visiongerencial/article/view/8192 consulta: 03/06/2017.

Dematteis, Giuseppe y Governa, Francesca (2005). Territorio y Territorialidad en el desarrollo local. La contribución del modelo SLOT. En: Boletín de la Asociación de Geógrafos Españoles. N 39 - 2005, España. (Pp. 31-58). Extraído de https://dialnet.unirioja.es/descarga/articulo/1161234.pdf consulta: 23/09/2015. 
Díaz, Zulay (2007). J. Habermas: Lenguaje y diálogo, el rol del entendimiento intersubjetivo en la sociedad moderna. En: Revista Utopía y Praxis Latinoamericana. Volumen $12 \mathrm{~N}^{\circ}$ 39, Venezuela. (Pp. 47-72). Extraído de http://www.redalyc.org/pdf/279/27903904.pdf consulta: 15/08/2010.

Fernández, $\quad$ Oscar $\quad$ (2008). Pierre $\quad$ Bourdieu:¿Agente oActor?Pierre_BourdieuBLOG.Extraído dehttp://pierrebourdieu.blogspot.com/2008/02/pierre-bourdieu-agente-o-actor-oscar.html consulta: 18/10/2017.

Gardner, Howard (2011). Inteligencias Múltiples. Editorial Paidós Ibérica. España.

García, Ester (2007). El concepto de actor. Reflexiones y propuestas para la ciencia política. En Revista Andamios, Volumen 3, Nº.6. Junio. México. Extraído de http://www.scielo.org.mx/scielo.php?script=sci_arttext\&pid=S187000632007000100008 Consulta 19/10/2017.

Habermas, Jürgen(2003). Acción comunicativa y razón sin trascendencia. Paidós. España.

Hurtado, Jacqueline (2000). Metodología de la Investigación Holística. Editorial Asociada Instituto Universitario de Tecnología Caripito y Fundación Sypal. Venezuela.

Jodelet, Denise (1986). La representación social: fenómenos, concepto y teoría. Paidós. España.

Morin, Edgar (2002). Los siete saberes necesarios para la educación del futuro. Editorial Paidos Ibérica. España.

Morin, Edgar (2009). Introducción al pensamiento complejo. Editorial Gedisa. España.

Organización de Estados Americanos (2011). Desigualdad e Inclusión en las Américas. Documentos Oficiales de la OEA. EEUU. Extraído de https://www.oas.org/docs/desigualdad/libro-desigualdad.pdf consulta: 23/01/2012.

Patiño Roberto y Varnagy Daniel (2012). Metodología para la medición del capital social en estudiantes de escuelas públicas de escuelas de Educación Básica y Educación Media. En Revista Politeia, Volumen 35, No 49. Universidad Simón Bolívar, Venezuela. (Pp. 99-157).Extraído de http://www.redalyc.org/pdf/1700/170029498005.pdf consulta: 15/12/2014.

Pozzoli, María (2006). Sujeto de la Complejidad. La construcción de un Modelo Teórico Transdisciplinar (eco-psico-socio-histórico-educativo). En: Revista Digital POLIS, Revista Latinoamericana, Volumen 15-2006, №5. Universidad de Los Lagos, Chile. (Pp. 25-55). Extraído de https://polis.revues.org/4921 consulta: 29/11/2016.

Soto González, Mario (1999). Edgar Morín: Complejidad y Sujeto Humano. Tesis Doctoral. Doctorado en Filosofía. Universidad de Valladolid. España. 
Extraído dehttp://www.biblioteca.org.ar/libros/133208.pdf consulta: $15 / 12 / 2016$.

UNESCO-ONU (2016). Informe Mundial sobre las Ciencias Sociales2016.Publicaciones ONU. Francia. Extraído dehttp://es.unesco.org/wssr2016 consulta: 12/12/2016

Vázquez-Barquero, Antonio (2007). Desarrollo endógeno. Teorías y políticas de desarrollo territorial. Ediciones Investigaciones Regionales. España.

Villafuerte, Luis (2008). Una metodología interpretativa para el estudio de los movimientos sociales. Enmarcamiento y cultura: Una Visión desde México. En: Revista Historia de la Educación Latinoamericana, Vol. 11, 2008. Universidad Pedagógica y Tecnológica de Colombia, Colombia. (Pp. 225246). Extraído dehttp://www.redalyc.org/pdf/869/86912618012.pdf consulta: 23/06/2010.

Villasante, Tomas, Montañés Manuel y Martí Joel (2000). La Investigación Social Participativa: Construyendo Ciudadanía. Editorial El Viejo Topo. España. 